\title{
Second Life as a Research Environment: Avatar-based Focus Groups (AFG)
}

\section{Introduction}

In recent years, rapid business growth on Virtual Worlds (VWs) ${ }^{1}$ attracted retailers to expand their market and improve their customer experience (Wasko et al. 2011). VWs simulate both the laws of physics as well as economic systems, providing valuable prospects for retailers (Bloomfield 2009; Jin 2009; Eisenbeiss et al. 2011). Yet, and despite the increased number of marketing research agencies specialising in conducting research on VWs, the suitability of VWs as a research tool in academic research is underdeveloped. This paper examines the nature and the potential of avatar-based focus groups (AFGs) ${ }^{2}$ (i.e., focus groups conducted in 3D VWs) as a marketing research tool, describing some of their characteristics, advantages, and limitations. Using a multi-reflective approach informed by the authors' experiences, the central components of this study are observation, comparison and reflection on data collected in two phases. This study advances our understanding of the potential use of AFGs and aims to encourage marketing researchers to use AFGs by discussing three issues: data quality, conduct of AFGs (including the moderator reflection) and participant experience.

\footnotetext{
${ }^{1}$ Virtual Worlds (VWs)

${ }^{2}$ Avatar-based Focus Groups (AFGs)
} 


\section{Virtual Worlds as a Marketing Research Tool}

According to Murgado-Armenteros, Torres-Ruiz and Vega-Zamora (2012, p. 74), focus groups are "the most commonly-used (qualitative) method in the area of market research." Evidence of the effectiveness of this method and the centrality of interaction to its success is well-established (e.g., Kitzinger 1994; Krueger and Casey 2000); yet, as with any method, critique can be made, especially in relation to the effects of power dynamics, fears about privacy, and some restrictions on sampling. The introduction of online focus groups (OFGs) ${ }^{3}$ (i.e., 2D text-based online chat websites) for example, data from online communication have been viewed as a less accurate reflection of thoughts than verbal data. Hodder (1994) argues that nonverbal observation is severely limited, lessening the role of the moderator, thereby reducing group dynamics and involvement. Although numerous studies show attempts to improve the effectiveness of OFGs (e.g., Montoya-Weiss, Massey and Clapper 1998; Bickart and Schindler, 2001), some problems are embedded into the nature of the online 2D environment and so are unlikely to significantly improve. With the limitations of 2D OFGs in mind, VWs promise to continue the evolutionary process started by the Web and the browser technology by maintaining most of the advantages of OFGs whilst compensating for some of the limitations through offering different stimuli and dynamics.

A VW is a computer-generated, multi-user, three-dimensional interface in which each user interacts freely with the environment through his or her individual avatar (Schroeder, 2002). 'Avatars' are defined as "general graphic representations that are personified by means of computer technology" (Holzwarth, Janiszewski, and Neumann,2006, p. 20). There are reasons to believe that AFGs held in VWs, where participants are present in the form of their avatar, can

${ }^{3}$ Online Focus Groups (OFGs) 
improve on the 2D OFGs. For example, existing research in the online 2D context indicates the positive impact of avatars (e.g., Ikea with Anna, Microsoft with Ms Dewey) to increase consumer interaction, provide entertainment value and ensure more personalised service (Holzwarth et al. 2006; Wang et al. 2007; McGoldrick, Keeling and Beatty 2008).

The most substantial arguments for the suitability of VWs as a research tool are based on Bloomfield's (2009) proposal that VWs simulate both the laws of physics as well as social and economic systems. Our reasoning is as follows: first, a VW platform allows for synchronous (or real-time) communication; this direct form of interaction enables interaction akin to physical real-life situations. Second, Holzwarth et al. (2006) concluded that the presence of a suitable avatar during the interaction enhances the perception of reciprocity and friendliness within the context of commercial 2D websites. Also, substantial research concludes that social, psychophysical and economic behaviours enacted in VWs are consistent with real life behaviours (e.g., Miller 2007; Chesney, Chuah and Hoffmann 2009). In an attempt to establish the reliability of VW's research, Massara et al. (2009) based their research on social response theory (Moon, 2000; Moon, 2003), indicating that research conducted in VWs produces similar patterns of results to research conducted in the real world but with the advantage of increased efficiency and control.

Third, according to the media richness theory (Daft and Lengel 1986), the richer the media format, the more effective the communication is in completing a task. Although the richest medium is face-to-face communications, 3D VWs are much richer than online 2D websites (Massara and Novak 2008) and can provide additional features and affordances (Park et al. 2008, Nah et al. 2011) owing to their specific characteristics. Compared to 2D websites, avatar presence provides a focus for social interaction, and helps engender immersion, high mutual 
awareness and social facilitation (Gerhard, Moore and Hobbs 2004). Moreover, users in the form of avatars with the support of the surrounding environment co-create their own experience; this stimulates their sense of presence leading to a more enjoyable virtual experience. Given these characteristics, VWs are developing into complex social systems through empowering an immersive human-computer interaction beyond the 2D text-based online chat websites (Massara and Novak 2008).

Fourth, proprietary software 3D platforms are starting to be very popular. One example is "The Clickroom" (http://www.theclickroom.com), an AFG tool that creates an interactive 3D environment using avatars to represent and engage with participants. From the information and endorsements on the website, international companies using this platform believe it has proved efficient in data collection over a broad demographic. Fifth, research conducted in VWs provides researchers with other advantages over both traditional studies (cost advantages) and pure webbased studies (control advantages). Generally, research in VWs overcomes the trade-off between the efficiency of online surveys and the effectiveness of traditional research, qualifying as a possible innovation in the social sciences (Bainbridge, 2007).

To sum up, Chesney et al. (2009) concluded that VWs can simulate most of the crucial features of a physical laboratory at much lesser cost. Due to this consistency across real and virtual environments, VWs can serve as laboratory environments for furthering consumer knowledge (Hemp 2006; Novak 2010) and more generally can serve as a platform for scientific research (Miller 2007; Bainbridge 2007). Moreover, because of the relative novelty of such environments, more evidence is necessary to investigate the applicability of using Second Life as a focus group research environment and to shed greater light on how to make the most of this opportunity. 


\section{Conduct of Research}

The VW platform for these studies is Second Life (SL), arguably considered the leading and largest VW (Jung and Pawlowski 2014). This platform is chosen because of the ease of access and cost efficiencies compared to proprietary virtual platforms (e.g., the clickroom platform), though the latter may provide some advantages in respondent recruitment. Due to the unique characteristics of the 3D context and the limited available literature on $\mathrm{AFG}$, it was important to use a multi-reflection approach. Central components of this study are observation, comparison and reflection on data collected in two phases. For the first phase, involving observation and comparison of face-to-face focus groups and AFG, we agree with Fox, Morris and Rumsey (2007, p. 539), that a reflective approach is most useful for identifying key challenges and stages associated with the methodological approach used and helps provide a pragmatic but useful account of the method. Thus, our analysis is informed by the reflective approach (Fox, Morris and Rumsey 2007; Ben-Ari and Enosh 2011) in that we describe and reflect on the first, observational stage comparing face-to-face focus groups and AFGs to identify significant advantages and challenges in AFG use. The reflection at this phase is centred on three perspectives, data quality, conduct of AFGs, and the moderator's reflection.

In phase 2, an online semi-structured survey provided comparison data and opinions from participants on their participation experiences in AFGs, 2D OFGs and traditional face-to-face focus groups. This phase offered a descriptive reflective discussion on participants' experience during AFGs and their differing viewpoints on the effectiveness of AFGs. This combination of methods and perspectives informs and enhances existing contributions on the VW as a research platform by examining how the 3D virtual context influences focus group discussion and 
dynamics. We present evidence to promote the AFGs as a way to overcome some of the limitations typically found in OFGs.

\section{Phase 1: Face-to-face focus groups versus AFGs}

We conducted three traditional face-to-face focus groups followed by three AFGs in SL (60-90 minutes duration). Figure 1 shows one actual AFG conducted by this study. Participant recruitment for these AFGs through a marketing research agency ensured a purposive sample reflecting SL demographics. The topic (factors affecting virtual shopping), explanation of the purpose, and consent procedures were identical for both contexts. Respondents for both contexts

were 'users' of a virtual store in Second Life; defined as a person who has visited, purchased from, or enjoyed the services offered by a virtual store at least once in the last three months. 
Figure 1 AFGs at Second Life

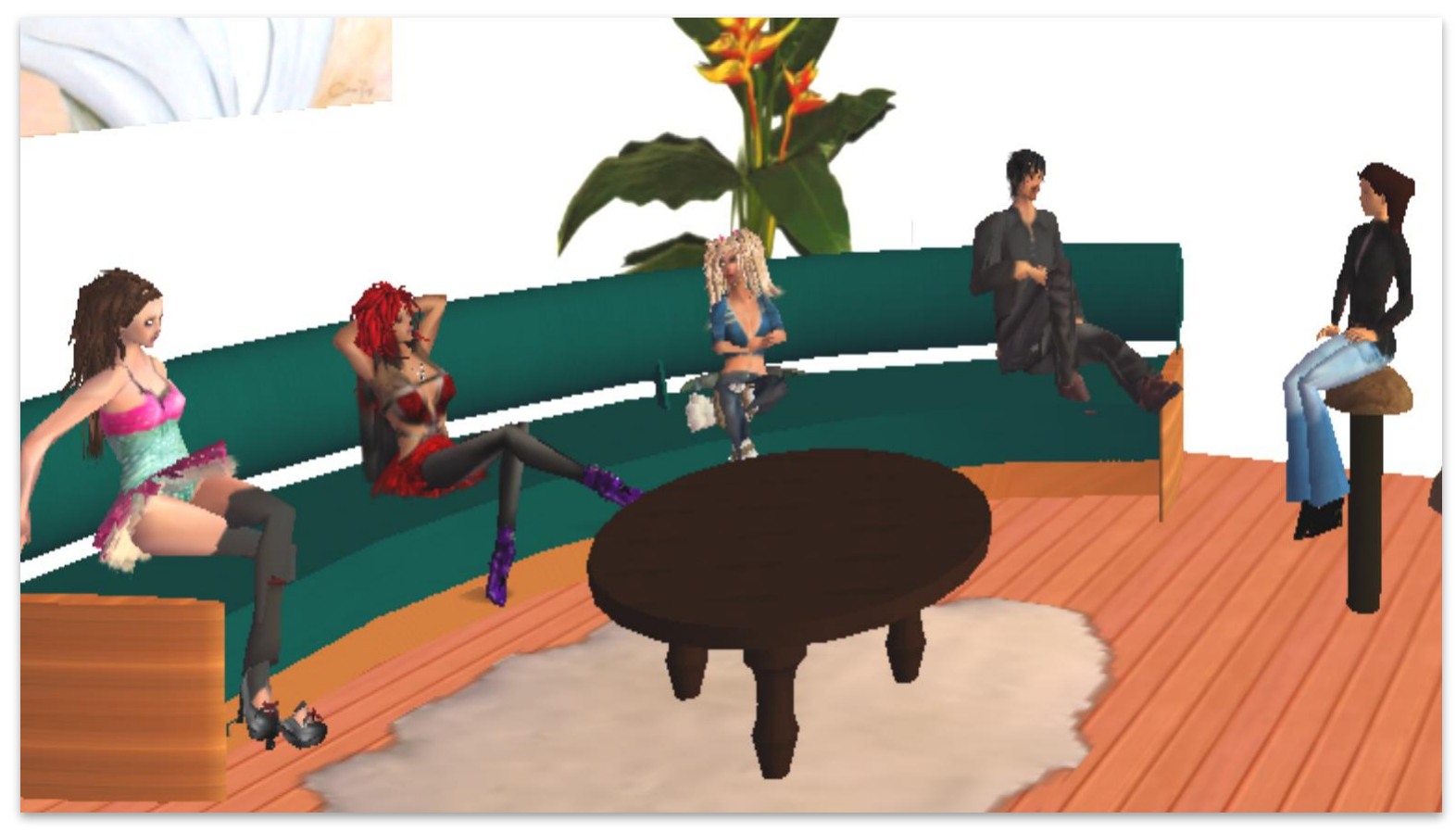

We reflect on our own observations and experience on conducting AFGs from three perspectives.

\section{Perspective 1: Data quality}

Table 1 gives detailed information on the focus groups conducted including the number of factors identified and total words recorded. Comparison of focus group outcomes from the traditional face-to-face and 3D context, but discussing the same topic, shows parallel patterns on various issues discussed. Participants in both types of focus groups offered approximately similar information. The volume of responses in the face-to-face focus groups comprised a mean total of 3208 words per group, compared to a mean of 3912 words for AFGs. This challenges the notion of more detailed discussion and expression in physically face-to-face focus groups compared to 
the physically removed AFGs. Participants in both types of focus group were interactive and expressed their opinions equally informatively and meaningfully, evidenced by the number of factors identified in each focus group: 42 (average 14) codes from the face-to-face focus groups versus 40 (average 13.3) from AFGs. The use of qualitative description, illustration and examples were equally rich within both types of focus groups. However, emergent themes via AFG were more direct and straightforward, perhaps because the topic of research was related to shopping in VWs, and conducting the focus groups within the same environment allowed the participants to reflect on their experiences in a more precise and expressive manner.

Table 1: Comparison between traditional groups and AFGs

\begin{tabular}{|c|c|c|c|c|c|}
\hline $\begin{array}{l}\text { Face-to-Face } \\
\text { Focus Groups }\end{array}$ & $\begin{array}{l}\text { Identified } \\
\text { factors }\end{array}$ & $\begin{array}{l}\text { Total } \\
\text { words }\end{array}$ & $\begin{array}{l}\text { Avatar-based } \\
\text { Focus Groups }\end{array}$ & $\begin{array}{l}\text { Identified } \\
\text { factors }\end{array}$ & Total words \\
\hline Focus Group 1 & 16 & 4068 & Focus Group 4 & 15 & 5311 \\
\hline Focus Group 2 & 11 & 2001 & Focus Group 5 & 13 & 2617 \\
\hline Focus Group 3 & 15 & 3556 & Focus Group 6 & 12 & 3809 \\
\hline Total & 42 & 9625 & Total & 40 & 11737 \\
\hline Mean & 14 & 3208.33 & Mean & 13.33 & 3912.33 \\
\hline
\end{tabular}

Both focus groups types showed similar levels of immediacy and depth. However, while it is easier in traditional face-to-face focus groups to see detailed facial expressions, avatar visual expression is less clear. Thus, the slight increase in the number of words used in AFGs may be due to participant attempts to compensate for the reduced facial and body expression during discussion. Accompanying this, there was explicit use of emoticons and other methods of showing emphasis and emotion (see observation 6 below). 


\section{Phase 1: Perspective 2: The conduct of AFG}

Using direct observation, the second perspective reflects on the conduct of AFGs through description of significant events and their implications for the nature and dynamics of AFG. The review of each of seven noteworthy observations is followed immediately by two key reflections: 1) the benefits for using the AFG method, 2) the challenges that the observed behaviour may pose for using AFGs or that may impact the quality of the collected data.

Observation 1: One participant was 10 minutes late for the AFG because she felt there was not a good enough outfit to wear in her 'inventory' so went shopping before the FG as she "wanted to feel good". Indeed, the new dress was impressive in some respects that are difficult for digital clothes. Her late entrance allowed others to notice these features as she entered the FG room, they were impressed by her look, asking where the dress came from although the participants did not know each other. Two asked if they could go shopping with her later.

- Reflection on the benefits: This situation eases the role of the moderator in breaking the ice plus it was easy for participants to bond quickly with little interference from the moderator. Additionally, the relative anonymity may allow respondents to be more socially interactive than might be the case in physical proximity.

- Reflection on the challenges: Users care about their appearance as they do in real life; this can be a source of distraction where participants are concerned with their appearance but this can be controlled by the moderator. 
Observation 2: One participant kept logging off and on due to a technical problem. So, we used the snowballing technique and asked the available participants if they had a friend willing to participate. In just a matter of minutes, the group was complete and discussion started.

- Reflection on the benefits: A benefit of the growing online community. Compared to the face-to-face situation, where if one participant is unable to make it there is no chance to solve the problem, in the online context the problem can be solved due to increased access to people. Thus, organisers can avoid the usual remedy of over-recruitment, or at least to have extra recruits standing by rather than having to pay for un-needed recruits.

- Reflection on the challenges: Technical problems can sometimes affect participation in general or the flow of conversation if the respondent cannot maintain contact.

Observation 3: One respondent attended as an avatar in the form of an antique clock, another as a fairy, yet another as a white cloud. The other participants showed respect to the person whose avatar was the self-designed clock, as this involves a lot of technicalities.

- Reflection on the benefits: The appearance of avatars is important and valued by other avatars, similar to appearances in real life (i.e., treating VWs as a real-life situation). Where identity and appearance are important to the discussion, these issues are accentuated by the ability of people to change avatar appearance and clothing easily and with little cost.

- Reflection on the challenges: This triggered off-topic discussions and also re-introduced some respondent hierarchy (albeit on grounds of technical ability) that is otherwise avoided when people can withhold identities behind avatars. 
Observation 4: A white cloud avatar revealed himself as an expert in 3D worlds. When participants knew the level of his expertise they started to ask him about certain technical problems.

- Reflection on the benefits: The use of avatars allows for both a friendlier environment and the metaphoric and symbolic aspects of appearance send signals allowing participants to easily connect with each other.

- Reflection on the challenges: This triggered off-topic discussions.

Observation 5: The same white cloud avatar was solving technical problems for clients during the focus group.

- Reflection on the challenges: The environment allows for multi-tasking, which can be a source of distraction (as in 2D OFGs). In this case, the participant was involved in the discussion and fully answered all questions. This might work because of his high level of expertise.

Observation 6: The use of symbols to convey emotions and feelings were evident in AFGs. Other actions such as nodding, thinking, giggling also expressed within the discussion indicate the participant is passionate about the topic or stressing the importance of certain factors.

- Reflection on the benefits: As in OFGs, this helps the researcher judge group mood and aid in richer interpretation of group dynamics, processes and meaning.

- Reflection on the challenges: Missing the body language of the other participants. 
Observation 7: One participant asked us for a short break as she wanted to check a cake in the oven. She disappeared for three minutes and during this time participants started to talk about the flexible nature of VWs.

- Reflection on the benefits: This reflects the friendly environment and how participants feel at home and comfortable, as the SL room context is not considered a formal environment. It is also an indication of the convenience of the method for participants.

- Reflection on the challenges: A source of distraction, if not controlled well by the moderator.

\section{Phase 1: Perspective 3: Moderator reflections on conducting AFGs}

The moderator reflections on conducting AFGs include some comparison with face-to-face focus groups as well as observations on some unique aspects of AFGs. First, developing rapport in AFGs is a relatively comfortable task for the moderator, participants engaged with each other quickly and in a very friendly manner with less effort from the moderator compared to face-toface focus groups.

Second, the role of the moderator involved more than asking and stirring the discussion: careful attention had to be paid to limit the off-topic comments and discussions, more so than with face-to-face focus groups. Fortunately, during these AFGs, off-topic comments did not sidetrack the main discussion as participants were chatting about specific virtual shopping experiences, which were still linked to the main topic under investigation, and this resulted in more informative comments and improved interaction. In some instances, interference to stop the off-topic discussion was unnecessary as it only lasted for a couple of minutes. Hence, off-topic comments were in fact short; for example, when discussion was centred on comparison between shopping in real life and SL, one of the off-topic comments related to how SL is beneficial in 
allowing people with special needs to do things that cannot be done in real life; one participant explained that she has a friend in real life who is fully paralysed and she is enjoying SL features such as flying and walking. This took only a couple of statements and without the moderator's interference to shift the discussion, the participant by herself apologised for being off-topic and the discussion continued. Therefore, the moderator must find the right balance between being considerate and empathetic and allowing for short off-topic comments without compromising the time available for the discussion. This example is also an illustration that all interactions can be treated as data, as this is informative about potential AFG advantages.

Third, some participants tended more to use symbols to convey their emotions and feelings. Although this might reflect different respondent personalities, these are useful in interpreting results; indeed, more so than voice inflection would be, as respondents are explicitly recording their emotions. So, the moderator introduction should expand to encourage participants to freely express their own emotions during the discussion.

Finally, during AFG, time seemed to pass very quickly compared to the face-to-face focus groups, possibly due to the interesting 3D context, which allows everyone to be comfortable in his/her own environment (i.e., whether at home, work, etc.), plus reading, keeping track of respondent text answers and replying to these. Hence, the moderator should carefully plan the topics for discussion to make sure that everything is covered during the allocated time of an AFG.

\section{Phase 2: AFG participants' reflections}

Conscious that a direct interview with respondents who already had a relationship with us could introduce some bias, and needing also respondents that had taken part in face-to-face, 2D online 
as well as AFG, data for this phase comes from three respondents, recruited through LinkedIn, who had participated in all three types of focus groups and so they could usefully be compared during completion of a semi-structured survey. Our aim was to maximise the amount of reflection and gain information from people that had participated in focus groups with other researchers and on other topics, even if this resulted in few but informative and detailed responses. Participants reflected on three areas: participants, the 3D virtual context, and the moderator of AFG.

\section{Participants}

Comments supported the concept that the online environment offers social equalisation as individual socio-economic status, ethnicity, nationality and gender may be unknown to other participants and can therefore serve as an unrestricted method of data collection. Anonymity can lessen inhibition and encourage freedom of speech, improving focus group data yield by facilitating more open and honest discussion (Montoya-Weiss et al. 1998; Reid and Reid 2005). This is evident in the following comments:

"Because of the protected and sharing environment, all interactions were open and equal."

"People can speak their opinions more freely." 


\section{D Virtual context: Medium effects}

Krueger and Casey (2000, p. 5) indicate the importance of conducting focus groups in a permissive, non-threatening environment. Respondent comments indicate that VWs such as SL can help successfully achieve such environments:

"Very relaxed and sharing group with no drama."

"One advantage is that it is a more comfortable environment for participants."

AFGs are highly interactive allowing participants to provide reflective, in-depth information. Additionally, the chat box enhances participant attention since the text appears prominently on the screen. Hence, participants have the time to reflect on others' opinions and think about their own responses:

"I like the way I wrote down my notes in the chat box and sent the message once the other participant finish talking, this helped me not to forget my points."

Interestingly, participants indicated that 3D representation (avatars) positively affected their experience; they enjoyed the focus groups and felt that they were fully participating. Moreover, when prompted about the possibility of the 3D representation as a source or distraction, they asserted that they were engaged and interacting and that the presence of avatars allowed them to bond quickly with other participants. This is a clear advantage of using AFG over 2D OFGs, where such immediate interaction and visualisation of identity are not possible (Murgado-Armenteros et al. 2012). Another advantage mentioned for VWs is reintroducing the ability to look at an object in 3D format; this adds to a positive experience and has an advantage over face-to-face in that it is easy to represent objects in 3D and in context that do not yet exist beyond the drawing board: 
"A lighting company produced a number of rooms in a house and a garden with new lighting concepts for respondents to wander around and try, whilst in the company of other focus group members. Then the group reconvened for a focus group meeting."

"A hotel group produced a number of rooms and respondents were encouraged to visit these to see and try out the facilities."

\section{The role of moderator}

When asked about their own participation, the importance of the role of the moderator was evident:

"It was good participation as it was fully moderated by a good facilitator."

"For me it was depending on the focus group facilitator who encouraged me to participate and not on the setting!"

Hence, as in other focus groups, the role and skills of the moderator are important in effectively and diligently managing the discussion:

"The moderator was not very good and did not explain things very well, so the discussion went off-track and lasted too long."

Moreover, another respondent indicated that the moderator as an avatar helps them to connect better and eliminate some of the disadvantages of face-to-face focus groups:

"This method can potentially remove the influence of the moderator [this referred to what can be felt as social pressure from the moderator] as you are not able to see his/her body language." 


\section{Discussion and Conclusion}

This study contributes to existing literature through advancing our understanding of the potential

use of AFG. Table 2 aggregates and presents advantages and disadvantages of AFG. In order to help researchers, these are grouped around three main elements: functional elements, technical elements, and methodological elements.

Table 2: Advantages/disadvantages of AFGs

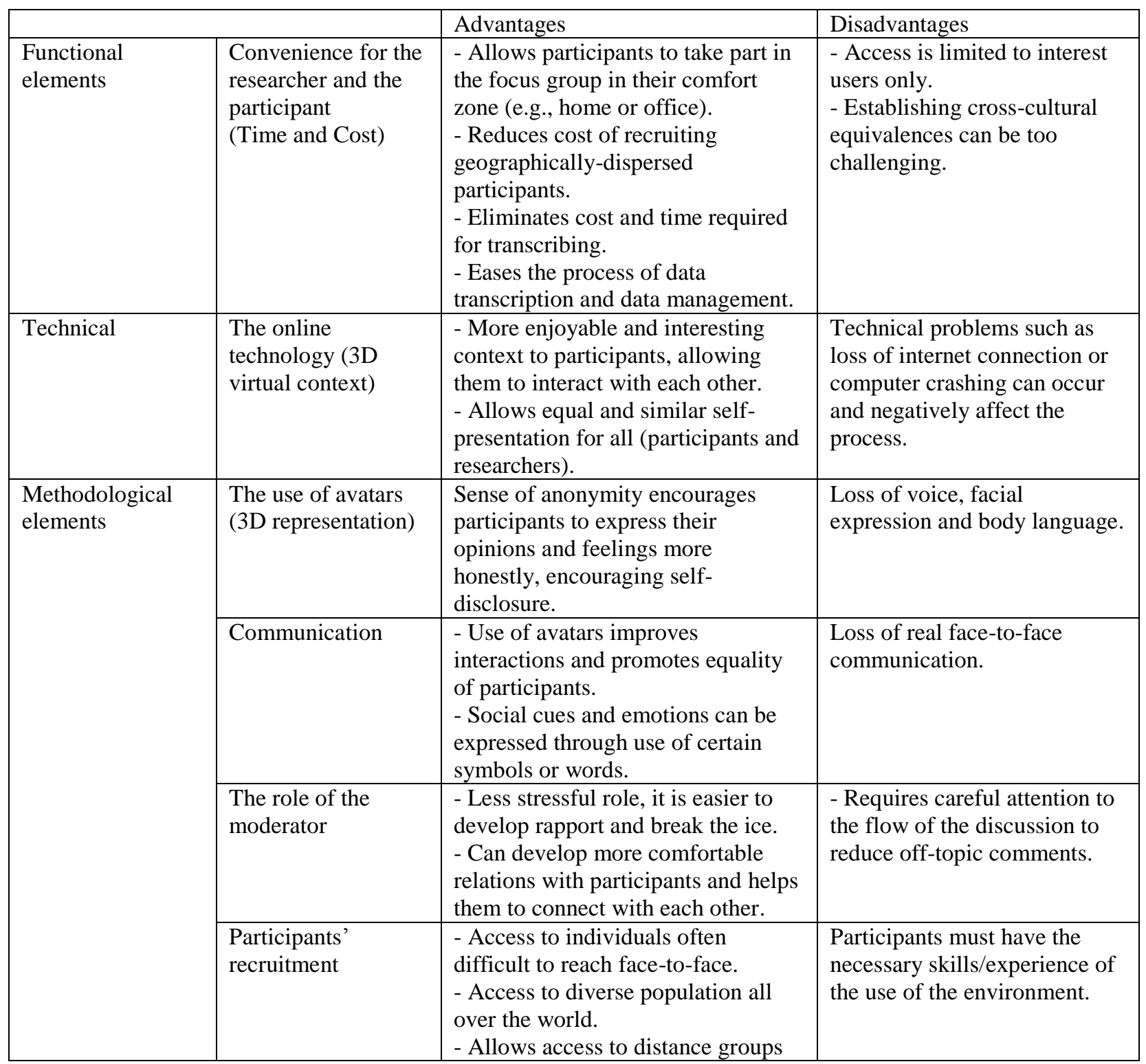




\begin{tabular}{|l|l|l|l|}
\hline & $\begin{array}{l}\text { normally difficult to recruit in } \\
\text { traditional FG. }\end{array}$ & \\
\hline
\end{tabular}

The findings from this study show that there might not be any evident differences in data quality between the results of avatar and face-to-face focus groups. This supports the view that VWs can imitate the conditions of the real world (Bloomfield, 2009, allowing for psychophysical, economic and social behaviours that mimic real-life behaviours (Bailenson et al. 2006; Miller, 2007 Chesney et al., 2009. Therefore, since social rules apply to general human-computer interaction, and since VWs provide such a vivid representation of the real world, we conclude that behaviour in AFGs approximates that in face-to-face focus groups.

We found compensation for lack of nonverbal (facial expression, body posture, etc.) and paraverbal cues (voice inflection, interjections, laughter, etc.). Consistent with Walston and Lissitz (2000) the use of common emotions and abbreviations can augment the lack of nonverbal and paraverbal cues in a virtual environment. We find analysis enhanced by these features as respondent feelings are explicit and not inferred. Accordingly, consistent with Massara et al. (2009), we believe that opinions provided in an AFG should not be less rich or detailed compared to those provided face-to-face, making the former an appropriate setting for marketing research.

This view highlights similarities associated with the use of AFGs versus face-to-face focus groups and OFGs. Participants in phase 2 explained this resemblance as follows: first, similar to OFGs, convenience for the researcher and the participant is one of the main advantages. AFGs are less expensive to operate than face-to-face focus groups and more appealing to both participants and researchers as there is no need to travel or leave their home or office. Having this freedom may convince people to participate, especially those who normally would not due to work-related scheduling difficulties or have difficulty with social confidence, 
travelling, or leaving home. Additionally, there is no need to manually transcribe the session and this also saves time and effort for the researcher. Second, the 3D context is considered more enjoyable than OFG and less stressful than face-to-face focus groups. The presence of avatars in SL compensates for the loss of face-to-face interaction in OFG, so we agree with Schroeder's (1997) view that "the combined effect of using text, navigating in a 3D environment and engaging with others via avatars ... is greater than the sum of its parts" (1997, p. 8). Consistent with Stewart and Williams (2005), the 3D virtual context boosts the feeling of 'presence', both visually through the surrounding context and the $3 \mathrm{D}$ representation of the avatar and textually through communicating with other avatars; this promotes more engaging discussions.

The use of the anonymous 3D representation in AFGs decreases social pressure and member dominance, allowing participants more perceived freedom to disagree with the moderator and/or other members. The $3 \mathrm{D}$ context has the potential to represent a neutral unbiased ground and provide participants with the opportunity to know each other and collaborate as a group in discussion without focusing on their perceived differences. This is consistent with existing research on OFGs (Montoya-Weiss et al. 1998; Schneider et al. 2002). Nonetheless, there are indications that the context may not be as unbiased as hoped: three observations on the conduct of AFGs suggest that some hierarchy and dominance could be introduced through avatar use.

Although this study advances existing knowledge on AFG, there is still much work required to gain a more complete understanding. Due to the distinctive features of VWs (e.g., anonymity with sense of immersion), future research should focus on the potential of AFG to collect in-depth data regarding sensitive topics related to health problems, addictive behaviour or collecting data from vulnerable consumers and/or children. In addition, future research needs to 
examine the use of AFG by different stakeholders on different VW platforms, e.g., between multinational firms, not-for-profit and academic researchers on both closed and open VWs. It appears that some multinational firms have been using AFG in proprietary platforms for its effectiveness and so future studies may compare the use of AFG in this situation where avatars and the virtual environment are controlled and accessed through a commercial company compared to use of AFG in open VWs. The range of avatars that attended the AFG displayed both dominant culture and sub-culture influences (e.g., the metaphor of the 'cloud' used by an IT expert). Future research should examine how to best capture 'cultural moments' during AFG, especially when the avatars are designed by participants themselves and may reflect a subculture within the VW (e.g., the fairy avatar is part of a distinct SL group). This will make any interpretation or observation of avatars more challenging when using open VW platforms such as SL. Balancing this are a) the reduction in entry costs, b) access to the very broad range of people who are members of open VWs, c) the sense of being 'at ease' and familiarity with the environment that our respondents felt in the SL AFGs, d) relative control of the design of the environment, and e) access to a range of skilled 'builders' of VW environments if 3D representations of products/places are needed.

To conclude, AFG provides real advantages and compensates for some of the serious limitations associated with online-chat focus groups, moreover most AFG shortcomings are manageable (except obviously where there is lack of suitable technology in a given region). We believe that this fast-growing context provides a suitable environment for researchers to collect data and holds many advantages over 2D OFGs and even, sometimes, over face-to-face focus groups. 


\section{References}

Bailenson, J.N., Blascovich, J., Beall, A.C. and Noveck, B. (2006), “Courtroom Applications of Virtual Environments, Immersive Virtual Environments, and Collaborative Virtual Environments", Law \& Policy, 28, April, 249-270.

Bainbridge, W.S. (2007), “The Scientific Research Potential of Virtual Worlds”, Science, 317, $5837,472-476$.

Ben-Ari, A. and Enosh, G. (2011), "Processes of Reflectivity Knowledge Construction in Qualitative Research", Qualitative Social Work, 10, 2, 152-171.

Bickart, B. and Schindler, R. (2001), "Internet Forums as Influential Source of Customer Information", Journal if Interactive Marketing, 15, 3, 31-40.

Bloomfield, R. (2009), “World of Bizcraft”, Journal of Virtual Worlds Research, 2, 3, 3-35.

Chesney, T., Chuah, S. and Hoffmann, R. (2009), "Virtual World Experimentation: An Exploratory Study", Journal of Economic Behavior and Organization, 72, 1, 618-635.

Eisenbeiss, M., Blechschmidt, B., Backhaus, K. and Freund, P. A. (2011), “'The (Real) World Is Not Enough:" Motivational Drivers and User Behavior in Virtual Worlds", Journal of Interactive Marketing, 26, August, 4-20.

Daft, R.L. and Lengel, R.H. (1986), “Organizational Information Requirements, Media Richness and Structural Design”, Management Science, 32, 5, 554-571.

Fox, F., Morris, M. and Rumsey, N. (2007), "Doing Synchronous Online Focus Groups with Young People: Methodological Reflections”, Qualitative Health Research, 17, 4, 539_ 547. 
Gerhard, M., Moore D.J. and Hobbs, D. (2004), "Embodiment and Co-Presence in Collaborative Interfaces”, International Journal of Human Computer Studies, 61, 4, 453-480.

Hemp, P. (2006), “Avatar-Based Marketing,” Harvard Business Review, 84, 48-57.

Hodder, I. (1994), “The interpretation of documents and material culture”. In Denzin, N. K. and Lincoln, Y. S. (Eds.), Handbook of Qualitative Research, (393-402). Thousand Oaks, CA: Sage.

Holzwarth, M., Janiszewski, C. and Neumann, M.M. (2006), "The Influence of Avatars on Online Consumer Shopping Behaviour”, Journal of Marketing, 70, 19-36.

Jin, S. A. (2009), “The Roles of Modality Richness and Involvement in Shopping Behavior in 3D Virtual Stores”, Journal of Interactive Marketing, 23, 3, 234-246.

Jung, Y. and Pawlowski, S. D. (2014), "Understanding Consumption in Social Virtual Worlds: A Sensemaking Perspective on the Consumption of Virtual Goods", Journal of Business Research, in press, DOI: http://dx.doi.org/10.1016/j.jbusres.2014.01.002.

Kitzinger, J. (1994), “The Methodology of Focus Groups: The Importance of Interaction between Research Participants”, Sociology of Health and Illness, 16, 1, 103-121.

Krueger, R. and Casey, M. (2000), Focus Groups: A Practical Guide for Applied Research, $3^{\text {rd }}$ edn, Thousand Oaks, CA: Sage Publications.

Massara, F. and Novak, T. P. (2008), "Factors Affecting Response Accuracy in Virtual Worlds," Advances in Consumer Research 2008, San Francisco, CA, October, 23-26.

Massara, F., Ancarani, F., Costabile, M., Moirano, M. and Ricotta, F. (2009), “Are You Telling me the Truth? Authenticity of Responses in Virtual Worlds Research", 8th International Congress Marketing Trends, Paris, France, 1-17. 
McGoldrick, P.J., Keeling, K. and Beatty, S. (2008), “A Typology of Roles for Avatars in Online Retailing", Journal of Marketing Management, 24, 3/4, 433-461.

Miller, G. (2007), “The promise of Parallel Universes,” Science, 317, 5842, 1341-1343.

Montoya-Weiss, M., Massey, A. and Clapper, D. (1998), “On-Line Focus Groups: Conceptual Issues and a Research Tool”, European Journal of Marketing, 32, 7/8, 713-723.

Moon, Y. (2000), "Intimate Exchanges: Using Computers to Elicit Self-Disclosure from Consumers," Journal of Consumer Research, 36, March, 324-340.

Moon, Y. (2003), “Don't Blame the Computer: When Self-Disclosure Moderates the SelfServing Bias," Journal of Consumer Psychology, 13, 1\&2, 125-137.

Murgado-Armenteros, E.M., Torres-Ruiz, F. and Vega-Zamora, M. (2012), "Differences between Online and Face to Face Focus Groups, Viewed Through Two Approaches", Journal of Theoretical and Applied Electronic Commerce Research, 7, 2, 73-86.

Nah, F., Eschenbrenner, B. and DeWester, D. (2011), "Enhancing Brand Equity Through Flow and Telepresence: A Comparison of 2D and 3D Virtual Worlds", MIS Quarterly, 35, 3, $731-747$.

Novak, T. P. (2010), “Elab City: A Platform for Academic Research on Virtual Worlds,” Journal of Virtual Worlds Research, 3, 1, 3-33.

Ondrejka, C. R. (2007), “Aviators, Moguls, Fashionistas and Barons: Economics and Ownership in Second Life," available at http://ssrn.com/abstract=614663

Park, S., Nah, F., DeWester, D., Eschenbrenner, B. and Jeon, S. (2008), "Virtual World Affordances: Enhancing Brand Value", Journal of Virtual Worlds Research, 1, 2, 1-18. 
Reid, D. J., and Reid, F. J. (2005), "Online Focus Groups: An In-Depth Comparison of Computer Mediated and Conventional Focus Group Discussions", International Journal of Market Research, 47(2), 131-162.

Schneider, S.J., Kerwin, J., Frechtling, J. and Vivari, B.A. (2002), "Characteristics of the Discussion in Online and Face-to-Face Focus Groups", Social Science Computer Review, $20,1,31-42$.

Schroeder, R. (2002), "Social Interaction in Virtual Environments: Key Issues, Common Themes, and a Framework for Research". In Schroeder, R. (eds.), The Social Life of Avatars, London, UK: Springer, 1-18.

Schroeder, R. (1997), "Networked Worlds: Social Aspects of Multi-user Virtual Reality Technology", Sociological Research Online, 2, 4, available online: http://www.socresonline.org.uk/socresonline/2/4/5.html

Stewart, K. and Williams, M. (2005), "Researching Online Populations: The Use of Online Focus Groups for Social Research”, Qualitative Research, 5, 4, 395-416.

Walston, J. T. and Lissitz, R. W. (2000), “Computer-Mediated Focus Groups”, Evaluation Review, 24, 5, 457-483.

Wang, L. C., Baker, J., Wagner, J. A. and Wakefield, K. (2007), "Can a Retail Web Site be Social?", Journal of Marketing, 71, 3, 143-157.

Wasko, M., Teigland, R., Leidner, D. and Jarvenpaa, S. (2011), "Stepping into the Internet: New Ventures in Virtual Worlds," MIS Quarterly, 35, 3, 645-652. 